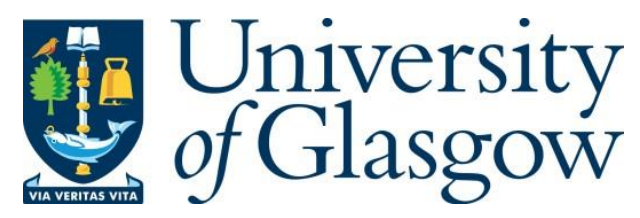

Brown, K.A. and Zhang, J.J. (2021) Survey on Hopf algebras of GK-dimension 1 and 2. Contemporary Mathematics, 771, pp. 43-62.

There may be differences between this version and the published version. You are advised to consult the publisher's version if you wish to cite from it.

http://eprints.gla.ac.uk/258159/

Deposited on: 2 November 2021

Enlighten - Research publications by members of the University of Glasgow http://eprints.gla.ac.uk 


\title{
SURVEY ON HOPF ALGEBRAS OF GK-DIMENSION 1 AND 2
}

\author{
K.A. BROWN AND J.J. ZHANG
}

\begin{abstract}
This is a brief survey on the current state of play on the programs to classify infinite dimensional Hopf algebras of Gel'fand-Kirillov dimension one or two. We list a number of open questions and suggest directions for future work.
\end{abstract}

\section{INTRODUCTION}

During the last 20 years, there has been significant progress towards a deeper understanding of infinite dimensional Hopf algebras satisfying some natural finiteness conditions. There has been wide interest in this effort, most notably in the programs concerning (both finite and infinite dimensional) Nichols algebras led by Andruskiewitsch, Angiono, Heckenberger, Schneider and others AA06, AS00, AS04, AAH19, Ang13, and the program concerning affine noetherian Hopf algebras initiated by Brown and Goodearl in 1997 BG97, Br98, BZ08, BZ10, LWZ07, WZ03, WLD16]. The subject of the present short survey is the classification and analysis of infinite dimensional Hopf algebras of low Gel'fand-Kirillov dimension. This topic lies at the intersection of the above two projects. Thus, as well as yielding results which are of interest in their own right, the research which we describe is a rich source both of examples and of methods which will prove useful in further work on both the above long-term endeavors.

The low-dimensional classification project has necessitated the development and refinement over the last 20 years of a number of algebraic concepts and homological invariants, for example AS-regularity, the Nakayama automorphism, the integral order and the integral minor, some of which we shall review here. These have played important roles in the further study of infinite dimensional noetherian Hopf algebras, strengthening connections to noncommutative algebraic geometry and the representation theory of quantum groups, with further applications likely in combinatorics, representation theory, statistical mechanics, topology and mathematical physics. To give one example, the project to classify prime AS-Gorenstein Hopf algebras of GK-dimension 1 in characteristic 0 has culminated in work of Liu Li20 which provides large families of examples ripe for exploitation in the study of tensor categories, support varieties, Hochschild cohomology, and so on. On the other hand despite (or rather, because of) this recent progress, very many open questions remain, many of which we have listed and discussed in what follows. For these reasons it is therefore a good time to briefly survey these classification programs.

Note that this account does not touch upon the large volume of recent significant research concerning finite dimensional (semisimple or not) Hopf algebras, or Nichols

2010 Mathematics Subject Classification. 16T05; Secondary 17B37,20G42.

Key words and phrases. Hopf algebra, Gel'fand-Kirillov dimension, homological integral. 
algebras, or tensor categories. For recent surveys on these topics we refer the reader to [And14, And17, EGNO15, respectively.

0.1 . General setup. Throughout, the base field will be denoted by $\mathbb{k}$ and will be assumed to be algebraically closed. For many of the results described this hypothesis can undoubtedly be weakened, but we will not pursue this question. On the other hand, the characteristic of the field will often be significant, so we will make clear the hypotheses concerning the characteristic of $\mathbb{k}$ on a case by case basis; whenever the characteristic is not mentioned, this means that the characteristic is arbitrary. All vector spaces, (co)algebras, tensor products, etc. are taken over $\mathbb{k}$. An algebra is called affine if it is finitely generated over $\mathbb{k}$. We refer to Montgomery's book Mo93 as a basic reference for Hopf algebras. For any Hopf algebra $H$, we denote the multiplication, unit map, comultiplication, counit and antipode by $m, u, \Delta, \epsilon$ and $S$ respectively. We use the same symbol $\mathbb{k}$ to denote both the trivial $\mathbb{k}$ algebra, and the trivial module over $H$, namely, $\mathbb{k}=H / \operatorname{ker} \epsilon$. Where not otherwise indicated, modules are left modules. Let $H^{o p}$ denote the opposite ring of $H$, so a right $H$-module can be viewed as a left $H^{o p}$-module and an $H$-bimodule is identified with a left $H \otimes H^{o p}$-module. Many examples and open questions on the topics discussed here can be found in textbooks and in the survey papers $\mathrm{Br} 98, \mathrm{Br} 07$, BGi14, Go13. In the present paper we will update these sources on their coverage of algebras of small GK-dimension, but we recommend them as references for wider issues concerning noetherian Hopf algebras.

0.2. Gel'fand-Kirillov dimension. Let $A$ be an algebra over $\mathbb{k}$. The Gel'fandKirillov dimension (or GK-dimension for short) of $A$ is defined to be

$$
\operatorname{GKdim} A:=\sup _{V} \limsup _{n \rightarrow \infty}\left(\log _{n}\left(\operatorname{dim}_{\mathbb{k}} V^{n}\right)\right)
$$

where $V$ runs over all finite dimensional subspaces of $A$. We refer to [KL00] for basic properties of GK-dimension of algebras and modules. Note that finite dimensional algebras have GK-dimension zero. Conversely, every algebra of non-zero GKdimension is clearly infinite dimensional. Examples of Borho-Kraft and Warfield KL00, Theorem 2.9], together with the Bergman Gap Theorem KL00, Theorem 2.5 ] show that GK-dimension of an affine algebra can take any value from the set

$$
\{0\} \cup\{1\} \cup[2, \infty] .
$$

The trichotomy (E0.0.1) motivates the study of Hopf $\mathbb{k}$-algebras of GK-dimension 1 and 2 , since these classes constitute a laboratory where classification is feasible and where more general conjectures can be tested. Note, however, that there is currently no known example of a Hopf algebra with finite non-integral GK-dimension. Thus we repeat [BGi14, Question F] (which was also asked by Zhuang [Zhu13]).

Question 0.1. BGi14, Question F] Does every Hopf algebra have either infinite or integral GK-dimension?

For further general questions on the GK-dimension of Hopf algebras, see BGi14.

0.3. Artin-Schelter and Cohen-Macaulay properties. The following concepts are key to the analysis of the Hopf algebras studied in this paper. Recall that a Hopf $\mathbb{k}$-algebra $H$ is Artin-Schelter Gorenstein (AS-Gorenstein for short) if

(AS1) injdim ${ }_{H} H=d<\infty$,

(AS2) $\operatorname{dim}_{\mathbb{k}} \operatorname{Ext}_{H}^{d}\left({ }_{H} \mathbb{k},,_{H} H\right)=1, \operatorname{Ext}_{H}^{i}\left({ }_{H} \mathbb{k}, H H\right)=0$ for all $i \neq d$, 
(AS3) the right $H$-module versions of the conditions (AS1), (AS2) hold.

We say $H$ is Artin-Schelter regular (AS-regular for short) if it is AS-Gorenstein and has finite global dimension; in this case gldim $H=d$. The homological grade of a non-zero $H$-module $M$ is defined to be $j(M):=\min \left\{j: \operatorname{Ext}_{H}^{j}(M, H) \neq 0\right\} \cup\{\infty\}$. Suppose that $H$ has finite GK-dimension. Then $H$ is GK-Cohen-Macaulay if, for all non-zero finitely generated $H$-modules $M$,

$$
j(M)+\mathrm{GK} \operatorname{dim} M=\mathrm{GK} \operatorname{dim} H .
$$

Notice that if $H$ is AS-Gorenstein and GK-Cohen-Macaulay then

$$
\operatorname{inj} \operatorname{dim} H=\mathrm{GKdim} H \text {. }
$$

All known noetherian Hopf algebras are AS-Gorenstein BZ08. A well-known conjecture in the study of noetherian Hopf algebras is the following, which was posted in [Br07, Question E] and [Go13, Question 3.5].

Conjecture 0.2 (Brown-Goodearl Conjecture). Every noetherian Hopf algebra is AS-Gorenstein.

We refer to $\mathrm{Br} 98, \mathrm{Br} 07, \mathrm{BZ08}, \mathrm{Go13}$, for further discussion of homological aspects of noetherian Hopf algebras.

0.4. Organization. The paper is organized as follows. In Section 1 we consider prime Hopf algebras of GK-dimension one, and in Section 2, we mainly consider Hopf algebra domains of GK-dimension two, with a digression to review Hopf Ore extensions. These two sections can be viewed as an expanded version of Go13, Section 4]. Section 3 contains a review and brief commentary on the many questions stated in Sections 0, 1 and 2.

\section{Hopf Algebras of GK-Dimension 1}

In this section we study Hopf algebras which are prime of GK-dimension one. By a famous result of Small and Warfield [SW84, when such an algebra (whether Hopf or not) is affine it is a finite module over its affine center, so it is noetherian and satisfies a polynomial identity. Thus if $H$ is a prime affine Hopf algebra of GKdimension 1, it is AS-Gorenstein and GK-Cohen-Macaulay by [WZ03, Theorems 0.1 and 0.2 . In particular, the injective dimension of $H$ (and its global dimension if $H$ is regular) are both 1 .

1.1. Preliminaries and key examples. Recall the classical result that the only connected algebraic groups of dimension one over an algebraically closed field $\mathbb{k}$ are the additive and multiplicative groups of $\mathbb{k}$, see for example, Hu75, Theorem 20.5] or [Bo91, Theorem III.10.9]. Translating to the language of Hopf algebras, the only affine commutative Hopf $\mathbb{k}$-algebra domains of GK-dimension 1 are $\mathbb{k}[X]$ and $\mathbb{k}\left[X^{ \pm 1}\right]$. But Tsen's theorem [Co77, p. 374] permits us to omit the commutative hypothesis, so combining [LWZ07, Corollary $7.8(\mathrm{a}) \Rightarrow(\mathrm{b})]$ with [GZ10, Proposition 2.1] yields the following.

Lemma 1.1. GZ10, Proposition 2.1(a,b)], BZ20, Lemma 5.1] The only affine Hopf $\mathbb{k}$-algebra domains of $G K$-dimension one are the coordinate rings $\mathbb{k}[X]$ and $\mathbb{k}\left[X^{ \pm 1}\right]$ of $(\mathbb{k},+)$ and $\left(\mathbb{k}^{\times}, \times\right)$respectively. 
Remark 1.2. In fact one can at least partly remove from Lemma 1.1 the hypothesis that $H$ is affine: Tsen's theorem still ensures that the algebras involved are commutative, so $S^{2}=$ Id by [Mo93, Corollary 1.5.12] and hence $H$ is a union of affine Hopf subalgebras. Let $H$ be a non-affine Hopf $\mathbb{k}$-algebra domain of GK-dimension one. The following statements follow from Lemma 1.1 .

(1) GZ10, Proposition 2.1(c)] Assume that $\mathbb{k}$ has characteristic 0. Then $H$ is a group algebra $\mathbb{k} G$ where $G$ is a non-cyclic torsion-free abelian group of rank one, i.e., a non-cyclic subgroup of $\mathbb{Q}$.

(2) When $\mathbb{k}$ has positive characteristic and $H$ contains a nontrivial grouplike element, then $H$ is a group algebra $\mathbb{k} G$ where $G$ is a non-cyclic torsion-free abelian group of rank one.

(3) If $H$ does not contain a nontrivial grouplike element, then $\mathbb{k}$ has positive characteristic, say $p$. If further $H$ is countably generated, then one can check that

$$
H=\mathbb{k}\left\langle X_{i}:\left[X_{i}, X_{j}\right]=0, X_{i}=F_{i}\left(X_{i+1}\right),(i, j \geq 1)\right\rangle,
$$

with $X_{i}$ primitive for all $i$, where $\left\{F_{i}(t)\right\}_{i \geq 1}$ is a sequence of polynomials of the form

$$
F_{i}(t)=\sum_{s \geq 0} \alpha_{i, s} t^{p^{s}}
$$

for some $\alpha_{i, s} \in \mathbb{k}$.

The following issue thus remains to be clarified.

Question 1.3. Let $\mathbb{k}$ have positive characteristic, and let $H$ be an uncountably generated Hopf domain over $\mathbb{k}$ of GK-dimension one. What is the structure of $H$ ?

For prime Hopf algebras of GK-dimension one which are not domains, the story is much more complicated and as yet incomplete even in characteristic 0 . We begin by recalling some key examples.

Example 1.4. Let $\mathbb{D}$ be the infinite Dihedral group $\left\langle g, x \mid g^{2}=1, g x g=x^{-1}\right\rangle$ and let $H$ be the group algebra $\mathbb{k} \mathbb{D}$. Since $\mathbb{D}$ has no nontrivial finite normal subgroups, $H$ is prime by Connell's theorem [Pas77, Theorem 4.2.10], and $H$ is regular if and only if $\mathbb{k}$ does not have characteristic 2, see Pas77, Theorem 10.3.13].

The second family is also well-known, sometimes called the infinite Taft algebras.

Example 1.5. Ta71, LWZ07, Examples 2.7 and 7.3] Let $n$ and $t$ be positive integers with $n \geq 2$ and $1 \leq t \leq n$, and let $\xi \in \mathbb{k}$ be a primitive $n$th root of 1 . Let $H:=H(n, t, \xi)$ be the $\mathbb{k}$-algebra generated by $x$ and $g$ subject to the relations

$$
g^{n}=1, \quad \text { and } \quad x g=\xi g x,
$$

so $H$ is not commutative. The coalgebra structure of $H$ is defined by

$$
\Delta(g)=g \otimes g, \quad \epsilon(g)=1
$$

and

$$
\Delta(x)=x \otimes 1+g^{t} \otimes x, \quad \epsilon(x)=0 .
$$

Thus $H$ is cocommutative if and only if $t=n$. The antipode $S$ of $H$ is defined by

$$
S(g)=g^{-1}, \quad \text { and } \quad S(x)=-g^{-t} x=-\xi^{t} x g^{-t} .
$$

Then $H$ is affine prime AS-regular of GK-dimension one, with center $Z(H)=\mathbb{k}\left[x^{n}\right]$. 
In 2009 Liu discovered a new family of Hopf algebras of GK-dimension one:

Example 1.6. [Li09, Proposition 2.1] Let $n \geq 2$ and $\xi \in \mathbb{k}$ be a primitive $n$th root of 1 . Let $A(n, \xi)$ be generated by $x, g$ and the inverse $g^{-1}$, with relations

$$
x g=\xi g x, \text { and } x^{n}=1-g^{n} .
$$

The coalgebra structure of $A(n, \xi)$ is defined by

$$
\Delta(g)=g \otimes g, \quad \epsilon(g)=1
$$

and

$$
\Delta(x)=x \otimes 1+g \otimes x, \quad \epsilon(x)=0 .
$$

Then $A(n, \xi)$ is affine prime AS-regular of GK-dimension one with center $\mathbb{k}\left[x^{n}\right]$.

1.2. Integral order and minor. The project to classify prime regular Hopf algebras was begun in [LWZ07. The homological integral was defined there - this has proved crucial for further analysis of AS-Gorenstein Hopf algebras, including classification projects. In this subsection we review the basic definitions, generalising the classical integrals of finite dimensional Hopf algebras [Mo93, Chapter 2].

Definition 1.7. [LWZ07, Definition 1.1] Let $H$ be an AS-Gorenstein Hopf algebra of injective dimension $d$. Any nonzero element in $\operatorname{Ext}_{H}^{d}\left({ }_{H} \mathbb{k}, H H\right)$ is called a left homological integral of $H$. Write $\int^{l}:=\operatorname{Ext}_{H}^{d}\left({ }_{H} \mathbb{k},,_{H} H\right)$. A nonzero element in $\int^{r}:=$ $\operatorname{Ext}_{H^{o p}}^{d}\left(\mathbb{k}_{H}, H_{H}\right)$ is called a right homological integral of $H$. By abuse of language we also call $\int^{l}$ and $\int^{r}$ the left and right homological integrals of $H$ respectively.

Notice that $\int^{l}$ is an $H$-bimodule of $\mathbb{k}$-dimension 1 , trivial as a left $H$-module but in general nontrivial on the right. For any 1-dimensional left (or right) $H$-module $M$, we can write it as $H / I$ for an ideal $I$ with $\operatorname{dim}_{\mathbb{k}} H / I=1$, or equivalently view it as an algebra homomorphism $\phi_{M}: H \rightarrow H / I$. Then $\phi:=\phi_{M}$ is a grouplike element in the dual Hopf algebra $H^{\circ}$, whose order is denoted by $o(M) \in \mathbb{N} \cup\{\infty\}$. Equivalently, $o(M)$ is the least positive integer $m$ such that $M^{\otimes m}$ is the trivial module $\mathbb{k}$; so $o(M)=1$ if and only if $M=\mathbb{k}$.

Continuing with the above notation, the left winding automorphism of $H$ associated to $\phi$ is defined to be

$$
\Xi_{\phi}^{l}: h \rightarrow \sum \phi\left(h_{1}\right) h_{2}, \quad \forall h \in H
$$

and the right winding automorphism associated to $\phi$ is

$$
\Xi_{\phi}^{r}: h \rightarrow \sum h_{1} \phi\left(h_{2}\right), \quad \forall h \in H
$$

Both $\Xi_{\phi}^{l}$ and $\Xi_{\phi}^{r}$ are in the group $\operatorname{Aut}_{a l g}(H)$ of algebra automorphisms of $H$. Let $G_{\phi}^{l}\left[\right.$ resp. $\left.G_{\phi}^{r}\right]$ (or $G_{M}^{l}\left[\right.$ resp. $\left.G_{M}^{r}\right]$ ) denote the subgroups of $\operatorname{Aut}_{a l g}(H)$ generated by $\Xi_{\phi}^{l}\left[\right.$ resp. $\left.\Xi_{\phi}^{r}\right]$.

Definition 1.8. Let $H$ be an AS-Gorenstein Hopf algebra.

(1) [LWZ07, Definition 2.2] The integral order of $H$ is

$$
i o(H):=o\left(\int^{l}\right)=\left|G_{{ }^{l}}^{l}\right| \in \mathbb{N} \cup\{\infty\},
$$

where $\int^{l}$ is considered as a right $H$-module.

(2) $H$ is called unimodular if $i o(H)=1$. 
(3) [BZ10, Definition 2.4] Suppose $i o(H)<\infty$. The integral minor of $H$ is

$$
i m(H):=\left|G_{\int^{l}}^{l} /\left(G_{\int^{l}}^{l} \cap G_{\int^{l}}^{r}\right)\right| .
$$

where the intersection is taken inside $\operatorname{Aut}_{a l g}(H)$.

By [LWZ07, Lemma 2.1], io $(H)$ equals $o\left(\int^{r}\right)$, viewing $\int^{r}$ as a left $H$-module. So it is not hard to show [LWZ07, BZ10] that

$$
i o(H)=\left|G_{f^{l}}^{r}\right|=\left|G_{\int^{r}}^{l}\right|=\left|G_{\int^{r}}^{r}\right| .
$$

Clearly $i m(H)$ divides $i o(H)$ if $i o(H)$ is finite. For the Taft algebras $H=H(n, t, \xi)$ of Example 1.5 it is not hard to check that $i o(H)=n$ and $i m(H)=n / \operatorname{gcd}(n, t)$, BZ10, page 273].

1.3. Classification results. The connection between the concepts just defined and classification was made clear in [LWZ07, with the following result.

Theorem 1.9. [LWZ07, Theorem 7.1] Let $H$ be an affine prime AS-regular Hopf algebra of $G K$-dimension one. Then io $(H)<\infty$ and it equals the PI-degree of $H$. As a consequence, $H$ is unimodular if and only if $H$ is commutative.

The commutative algebras which occur in Theorem [1.9 are listed in Lemma 1.1. Progress was achieved towards a classification of the noncommutative ones in characteristic 0 by analysing their integral order and minor. Here are the two key theorems from BZ10.

Theorem 1.10. BZ10, Theorem 4.1] Assume that $\mathbb{k}$ has characteristic 0, and let $H$ be an affine prime AS-regular Hopf $\mathbb{k}$-algebra of GK-dimension one. Suppose $i o(H)>1$ and $\operatorname{im}(H)=1$. Then $H$ is one of the following.

(1) $H$ is the Taft algebra $H(n, n, \xi)$ of Example 1.5. In this case $i o(H)=n$.

(2) $H$ is the Dihedral group algebra $\mathbb{k} \mathbb{D}$ of Example 1.4 . In this case $i o(H)=2$.

In both cases, $H$ is cocommutative.

The next result classifies all Hopf algebras in Theorem 1.9 with $i m(H)=i o(H)$. To save space we will not give the definition of the generalized Liu algebras, denoted by $B(n, w, \xi)$, see [BZ10, Section 3.4] for details. Here $\xi$ is a primitive $n$th root of 1 and $w$ is a positive integer. When $\operatorname{gcd}(n, w)=1$ one obtains precisely Liu's algebras from Example 1.6.

Theorem 1.11. [BZ10, Theorem 6.1] Assume that $\mathbb{k}$ has characteristic 0, and let $H$ be an affine prime AS-regular Hopf algebra of GK-dimension one. Suppose $i o(H)=i m(H)>1$. Then $H$ is one of the following.

(1) The Taft algebra $H(n, 1, \xi)$ of Example 1.5.

(2) The generalized Liu algebra $B(n, w, \xi)$.

In both cases, $i o(H)=n$ and $H$ is not cocommutative.

In BZ10, Question 7.1] we asked if, in characteristic 0, every Hopf algebra in Theorem 1.9 satisfies either $i m(H)=1$ or $i m(H)=i o(H)$. The remarkable construction and result of Wu, Liu and Ding WLD16 answers this question in the negative, and in doing so provides the complete classification of the Hopf algebras in Theorem 1.9 in the characteristic 0 case. The new class of Hopf algebras $D(m, d, \xi)$ is defined in [WLD16, Subsection 4.1], requiring a couple of pages, and 
the proof that $D(m, d, \xi)$ is a Hopf algebra occupies 10 pages of WLD16, Subsection 4.2]. Basic properties of $D(m, d, \xi)$ are described in WLD16, Subsection 4.3]; in particular $i o(D(m, d, \xi))=2 m$ and $\operatorname{im}(D(m, d, \xi))=m$ [WLD16, Lemma 4.5].

Theorem 1.12. WLD16, Theorem 8.3] Asume that $\mathbb{k}$ has characteristic 0, and let $H$ be an affine prime AS-regular Hopf algebra of GK-dimension one. Suppose $i o(H) \neq i m(H) \neq 1$. Then $H$ is isomorphic to $D(m, d, \xi)$ for some choice of $m, d, \xi$.

From Theorems 1.9, 1.10, 1.11 and 1.12 one sees that, when $\mathbb{k}$ has characteristic 0 and the Hopf $\mathbb{k}$-algebra $H$ is prime affine AS-regular of GK-dimension one,

$$
i o(H) / i m(H) \text { is either } 1,2 \text { or } i o(H) \text {. }
$$

By [WLD16, Proposition 4.9], $D(m, d, \xi)$ is not pointed, giving a negative answer to Go13, Question 2.16] and [BZ10, Section 0.5].

1.4. Further questions and comments on GK-dimension one. After the progress described in Subsection 1.3 it is natural to ask if one can achieve a classification extending that of Theorems 1.10, 1.12 by removing one or more of the hypotheses that $(A) \mathbb{k}$ has characteristic $0,(B) H$ is AS-regular or $(C) H$ is prime. We briefly consider each of these in turn.

(A) This may be the easiest of the three, but it has received minimal attention so far. For the sake of precision, we formulate a specific question:

Question 1.13. Let $\mathbb{k}$ have characteristic $p>0$. Classify the prime affine ASregular Hopf $\mathbb{k}$-algebras $H$ of GK-dimension 1 when (i) $p \nmid i o(H)$ and (ii) $p \mid i o(H)$.

It may be that - at least in case (i) - this requires nothing more than a careful check of the arguments used for the results of Subsection 1.3. Along similar lines, one should also consider what happens when $\mathbb{k}$ is not algebraically closed. Even in the commutative case, new examples occur, as noted in [LWZ07, Example 8.3].

(B) Consider now the AS-regularity hypothesis. it was known already from BZ10, Example 7.3] that even in characteristic 0 there exist prime affine Hopf algebras of GK-dimension 1 which are not regular. Liu [Li20] started a program to classify all prime affine non-regular Hopf algebras $H$ of GK-dimension one when $\mathbb{k}$ has characteristic 0 . By WZ03, despite the absence of regularity, such an algebra $H$ is still AS-Gorenstein, so homological integrals are defined and homological invariants such as the integral order and integral minor can continue to be used. Liu's strategy is to impose two hypotheses on $H$ which are known consequences of regularity. For example, one of the hypotheses is to assume the existence of a nontrivial onedimensional $\mathrm{H}$-module $M$, specifically a module $M$ such that $o(M)=\operatorname{PIdeg} H$, where $o(M)$ is as defined in Subsection 1.2. Assuming these two extra hypotheses Liu is able to obtain a beautiful classification in which many new Hopf algebras occur, these being what he calls fractional versions of some of the regular algebras. This leaves an obvious project:

Question 1.14. Classify prime affine Hopf $\mathbb{k}$-algebras of GK-dimension 1 in characteristic 0 without assuming Liu's two supplementary hypotheses.

$(C)$ What happens when the primeness hypothesis is omitted? Recall that an affine algebra of GK-dimension one satisfies a polynomial identity (PI), thanks to [SWW85. By [LWZ07, Lemma 5.3(b)], every noetherian affine AS-regular Hopf 
algebra satisfying a PI is a direct sum of prime rings. In view of this and considering known examples, $\mathrm{Lu}, \mathrm{Wu}$ and Zhang propose the following conjectural structure of a noetherian affine AS-regular Hopf $\mathbb{k}$-algebra $H$ of GK-dimension one. Such an algebra $H$ should fit into a short exact sequence

$$
0 \rightarrow H_{d i s} \rightarrow H \rightarrow H_{\text {conn }} \rightarrow 0
$$

where the connected component $H_{\text {conn }}$ is an AS-regular prime factor Hopf algebra of $H$ of GK-dimension 1, and the discrete component $H_{d i s}$ should be a finite dimensional subalgebra of $H$ with a braided Hopf algebra structure. In LWZ07, Theorem 6.5(b)], Lu, Wu and Zhang were able to prove much of this, but not the finite dimensionality of $H_{\text {dis }}$. We therefore ask:

Question 1.15. Is the above conjecture correct?

The answer to this question might well be important for the structure of AS-regular noetherian Hopf algebras which are not of GK-dimension 1, nor even PI.

In parallel with trying to answer Question 1.15, one should try to construct all possible algebras provided by the recipe of $\mathrm{Lu}$, Wu and Zhang. This philosophy motivates the following question.

Question 1.16 (Gongxiang Liu Li20a]). Let $\mathbb{k}$ have characteristic 0 and let $H$ be an affine prime AS-regular Hopf algebra of GK-dimension 1, as listed in Lemma 1.1 and Theorems 1.10, 1.11 and 1.12. Classify finite dimensional semisimple braided Hopf algebras in the Yetter-Drinfeld category ${ }_{H}^{H} \mathcal{Y} \mathcal{D}$. These objects should play the role of $H_{\text {dis }}$ in (E1.14.1).

1.5. The bridge from GK-dimension 1 to GK-dimension 2. As a step towards looking at prime affine Hopf algebras of GK-dimension 2 in the next section, we first note that, when $\mathbb{k}$ has characteristic 0 , many such algebras can be constructed as extensions of an (in general braided) Hopf algebra of GK-dimension 1 by a second such algebra. This is true in the commutative case, as is recalled in Subsection 2.1 below, and is also true in the cocommutative case, as can be deduced from the structure theorem of Cartier-Kostant-Gabrièl [Mo93, 5.6.4, 5.6.5]. Many more examples can be constructed from the algebras featuring in Subsection 1.3 by use of the tensor product, as in part (3) of the following result.

Proposition 1.17. Let $\mathbb{k}$ be an algebraically closed field of characteristic 0 , and let $H$ and $K$ be prime affine AS-regular Hopf $\mathbb{k}$-algebras of $G K$-dimension one. Let $n$ and $m$ be the integral orders of $H$ and $K$ respectively.

(1) The center $Z(H)$ of $H$ is isomorphic to either $\mathbb{k}[t]$ or $\mathbb{k}\left[t^{ \pm 1}\right]$.

(2) The Goldie quotient ring of $H$ is isomorphic to $M_{n}(\mathbb{k}(t))$.

(3) The tensor product $H \otimes K$ is a noetherian prime affine AS-regular Hopf algebra of GK-dimension 2. Moreover $H \otimes K$ is a free module of rank $n m$ over its center $Z(H) \otimes Z(K)$.

Proof. (1) This follows from the classification in Subsection 1.3. One can check the assertion directly for the algebras in Theorems 1.9, 1.10 and 1.11(1). For the generalized Liu algebra $B(n, w, \xi)$ in Theorem 1.11(2), the assertion follows from [BZ10, Theorem 3.4(b)]. For the algebras $D:=D(m, d, \xi)$ in Theorem 1.12, we claim that $Z(D)=\mathbb{k}[t]$ where $t=x+x^{-1}$. We outline the argument, freely using the notation introduced in WLD16. Indeed, the algebra $D$ has a bigrading $D=\bigoplus D_{i j}$ with details given in [WLD16, equation (4.7)]. From this bigrading 
it is not hard to see that $Z(D) \subseteq D_{00}$, where $D_{00}$ is exactly the Hopf subalgebra $\mathbb{k}\left[x^{ \pm 1}\right]$. Since $x u_{i}=u_{i} x^{-1}$ for $0 \leq i \leq m-1$ by the definition of $D(m, d, \xi)$, the center is contained in the subalgebra $\mathbb{k}\left[x+x^{-1}\right]$. Conversely, it is easy to check that $x+x^{-1}$ commutes with all generators of $D$.

(2) This follows from part (1), by [SW84] and Tsen's theorem [Co77, p. 374].

(3) First, $H$ and $K$ are finite over their affine centers. By the Künneth formula, we obtain that $H \otimes K$ has global dimension at most 2 (a similar argument was used in the proof of [LWZ09, Theorem 3.2]). As a consequence, $H \otimes K$ is a noetherian affine PI AS-regular Hopf algebra of GK-dimension two. By [BG97, Theorem A], $H \otimes K$ is a finite direct sum of prime rings.

We claim that $H \otimes K$ is prime. By $(2), Q(H) \cong M_{n}(\mathbb{k}(t))$ and $Q(K) \cong M_{m}(\mathbb{k}(s))$. Then $Q(H) \otimes Q(K) \cong M_{n m}(\mathbb{k}(s) \otimes \mathbb{k}(t))$, and hence

$$
Q(H \otimes K)=Q(Q(H) \otimes Q(K)) \cong M_{n m}(\mathbb{k}(s, t)) .
$$

This forces $H \otimes K$ to be prime by Goldie's theorem. Next, using (E1.17.1),

$$
Z(H) \otimes Z(K) \subseteq Z(H \otimes K) \subseteq Z(Q(H \otimes K))=\mathbb{k}(s, t)=Q(Z(H) \otimes Z(K)) .
$$

Note that $Z(H \otimes K)$ is a finite $Z(H) \otimes Z(K)$-module and thus integral over $Z(H) \otimes$ $Z(K)$. Since the latter algebra is integrally closed we deduce from (E1.17.2) that $Z(H \otimes K)=Z(H) \otimes Z(K)$. Finally, the freeness of $H \otimes K$ over this (possibly partly Laurent) polynomial subalgebra follows from the Cohen-Macaulay property WZ03, Theorems 0.1 and 0.2] and BM17, Theorem 3.7(ii)], and the rank from (E1.17.1) and (E1.17.2)

The following question, though at first glance rather technical, is suggested for the algebras $D(m, d, \xi)$ by considering the pattern followed by the other GKdimension one families, and is highly relevant to the classification program for GK-dimension two Hopf domains discussed in Section 2.

Question 1.18. Let $D(m, d, \xi)$ be the Hopf algebra in Theorem 1.12, Is there a noetherian affine Hopf domain $H$ of GK-dimension two containing either a grouplike element $g$ or a skew-primitive element $x$ with $H /\langle g-1\rangle \cong D(m, d, \xi)$ or $H /\langle x\rangle \cong$ $D(m, d, \xi)$ as Hopf algebras?

If such a Hopf algebra exists, it could not be pointed (since $D(m, d, \xi)$ is not) and so would demonstrate the existence of non-pointed affine Hopf domains in dimension 2. At the moment, the project to classify noetherian affine Hopf domains of GKdimension two only produces pointed Hopf algebras.

Note that the requirement that $H$ be a domain is key in Question 1.18 since $H=D(m, d, \xi)[t]$ with $t$ primitive or $H=D(m, d, \xi)\left[t^{ \pm 1}\right]$ with $t$ grouplike give easy prime examples as special cases of Proposition 1.17.

\section{Hopf AlGEBras of GK-DIMENSION 2}

In Section 2 we survey some results concerning Hopf algebra domains of GKdimension two. We continue to assume that $\mathbb{k}$ is algebraically closed, and - except in Subsections 2.3 and 2.6 - we assume that $\mathbb{k}$ has characteristic 0 . 
2.1. Commutative examples and dimension of tangent spaces. The tensor product recipe of Proposition 1.17 applied to the commutative Hopf algebras of Lemma 1.1 yields the following three commutative Hopf domains of GK-dimension two:

$$
\mathcal{O}(\mathbb{k})^{\otimes 2}, \mathcal{O}\left(\mathbb{k}^{\times}\right)^{\otimes 2}, \mathcal{O}(\mathbb{k}) \otimes \mathcal{O}\left(\mathbb{k}^{\times}\right) .
$$

The remaining affine commutative Hopf $\mathbb{k}$-algebra domains of GK-dimension two arise as follows.

Example 2.1. GZ10, Construction 1.1] Let $n$ be a positive integer. Let $A(n, 1)$ be the algebra generated by $x, x^{-1}, y$ subject to the relation $x y=y x$. There is a unique Hopf algebra structure on $A$ with comultiplication

$$
\Delta(x)=x \otimes x, \quad \Delta(y)=y \otimes 1+x^{n} \otimes y .
$$

Expressed in alternative language, examples (E2.1.0) and 2.1 give a list (which is of course well-known) of all connected two-dimensional affine algebraic groups over $\mathbb{k}$, as noted in [GZ10, Lemma 2.2].

In trying to classify all the affine Hopf domains of GK-dimension two it turns out to be both useful and natural to first impose an additional hypothesis. Namely we consider first such Hopf algebras $H$ for which

$$
\operatorname{Ext}_{H}^{1}(\mathbb{k}, \mathbb{k}) \neq 0 .
$$

Recall that if $A$ is any affine commutative $\mathbb{k}$-algebra of GK-dimension two and $\mathfrak{m}$ is a maximal ideal of $A$, then

$$
\operatorname{dim}_{\mathbb{k}} \mathfrak{m} / \mathfrak{m}^{2}=\operatorname{dim}_{\mathbb{k}} \operatorname{Ext}_{A}^{1}(A / \mathfrak{m}, A / \mathfrak{m}) \geq 2 .
$$

Indeed $\operatorname{dim}_{\mathbb{k}} \operatorname{Ext}_{A}^{1}(A / \mathfrak{m}, A / \mathfrak{m})$ is equal to the dimension of the tangent space at the point $\mathfrak{m}$ in Spec $A$, so it seems reasonable to consider the class of quantum groups $H$ for which the tangent space at any point of $H$ is nontrivial. In fact, as we record in the next lemma, E2.1.1 is equivalent to the condition that the corresponding quantum group contains a classical algebraic group of dimension 1 ; one might compare this with the analogous fact that every quantum projective plane in the sense of Artin and Schelter contains a classical commutative curve of dimension 1.

Lemma 2.2. GZ10, Theorem 3.9] Assume that $\mathbb{k}$ has characteristic 0 and let $H$ be a Hopf $\mathbb{k}$-algebra of GK-dimension at most two which is either affine or noetherian. Then $H$ satisfies (E2.1.1) if and only if $H$ has a Hopf quotient, denoted by $\bar{H}$, isomorphic to either $\mathbb{k}\left[t^{ \pm 1}\right]$ with $t$ grouplike or $\mathbb{k}[t]$ with $t$ primitive.

2.2. Noetherian Hopf domains of GK-dimension 2. Following Lemma 2.2 the classification of Hopf $\mathbb{k}$-algebras of GK-dimension 2 satisfying (E2.1.1) now splits into two cases: (i) $\bar{H}=\mathbb{k}\left[t^{ \pm 1}\right]$ or (ii) $\bar{H}=\mathbb{k}[t]$. The following result is due to Goodearl and Zhang GZ10. In it, families (I), (III) and (IV) constitute case (i), (II) and (V) case (ii).

Theorem 2.3. GZ10, Theorem 0.1] Let $\mathbb{k}$ be algebraically closed of characteristic 0 , and let $H$ be a Hopf $\mathbb{k}$-algebra domain of GK-dimension 2 satisfying (E2.1.1). Then $H$ is noetherian if and only if $H$ is affine if and only if $H$ is isomorphic to one of the following:

(I) The group algebra $\mathbb{k} \Gamma$, where $\Gamma$ is either 
(Ia) the free abelian group $\mathbb{Z}^{2}$, or

(Ib) the nontrivial semidirect product $\mathbb{Z} \rtimes \mathbb{Z}$.

(II) The enveloping algebra $U(\mathfrak{g})$, where $\mathfrak{g}$ is either

(IIa) the 2-dimensional abelian Lie algebra over $\mathbb{k}$, or

(IIb) the Lie algebra over $\mathbb{k}$ with basis $\{x, y\}$ and $[x, y]=y$.

(III) The Hopf algebras $A(n, q)$ in Example 2.4 below.

(IV) The Hopf algebras $B\left(n, p_{0}, \cdots, p_{s}, q\right)$ in Example 2.5 below.

(V) The Hopf algebras $C(n)$ in Example 2.6 below.

Example 2.4. GZ10, Construction 1.1] Let $n$ be a positive integer and $q \in \mathbb{k}^{\times}$, and define $A(n, q)$ to be the algebra $\mathbb{k}\left\langle x^{ \pm 1}, y \mid x y=q y x\right\rangle$. There is a unique Hopf algebra structure on $A(n, q)$ under which $x$ is grouplike and $y$ is skew primitive, with $\Delta(y)=y \otimes 1+x^{n} \otimes y$. Clearly Example 2.1 is the case $q=1$.

Example 2.5. GZ10, Construction 1.2] Let $n, p_{0}, p_{1}, \ldots, p_{s}$ be positive integers and $q \in \mathbb{k}^{\times}$with the following properties:

(a) $s \geq 2$ and $1<p_{1}<p_{2}<\cdots<p_{s}$;

(b) $p_{0} \mid n$ and $p_{0}, p_{1}, \ldots, p_{s}$ are pairwise relatively prime;

(c) $q$ is a primitive $\ell$ th root of unity, where $\ell=\left(n / p_{0}\right) p_{1} p_{2} \cdots p_{s}$.

Set $m=p_{1} p_{2} \cdots p_{s}$ and $m_{i}=\frac{m}{p_{i}}$ for $i=1, \ldots, s$. Fix an indeterminate $y$ and consider the subalgebra $A:=\mathbb{k}\left\langle y_{1}, \ldots, y_{s}\right\rangle \subseteq \mathbb{k}[y]$, where $y_{i}:=y^{m_{i}}$ for $i=1, \ldots, s$. The $\mathbb{k}$-algebra automorphism of $\mathbb{k}[y]$ sending $y \rightarrow q y$ restricts to an automorphism $\sigma$ of $A$. There is a unique Hopf algebra structure on the skew Laurent polynomial ring $B=A\left[x^{ \pm 1} ; \sigma\right]$ such that $x$ is grouplike and the $y_{i}$ are skew primitive, with

$$
\Delta\left(y_{i}\right)=y_{i} \otimes 1+x^{m_{i} n} \otimes y_{i}
$$

for $i=1, \ldots, s$. This Hopf algebra is denoted by $B\left(n, p_{0}, \ldots, p_{s}, q\right)$.

Example 2.6. GZ10, Construction 1.4] Let $n$ be a positive integer, and set $C(n):=\mathbb{k}\left[y^{ \pm 1}\right]\left[x ;\left(y^{n}-y\right) \frac{d}{d y}\right]$. That is, $C(n)$ is an Ore extension of $\mathbb{k}\left[y^{ \pm 1}\right]$ with indeterminate $x$ satisfying the equation

$$
x f(y)=f(y) x+\left(y^{n}-y\right) \frac{d f(y)}{d y}
$$

for all $f(y) \in \mathbb{k}\left[y^{ \pm 1}\right]$. There is a unique Hopf algebra structure on $C(n)$ such that $y$ is grouplike and $x$ is skew primitive, with

$$
\Delta(x)=x \otimes y^{n-1}+1 \otimes x .
$$

Naturally, in the light of Theorem 2.3 Goodearl and Zhang asked the obvious question: does the classification remain true without assuming (E2.1.1)? In 2013 Wang, Zhang and Zhuang WZZ13] showed that the answer is "No". We'll review their result in Subsection 2.5

Note that all the algebras listed in Theorem 2.3 are assembled from two onedimensional domains, as discussed in Subsection 1.5. In particular, $A(n, q)$ and $C(n)$ are Ore extensions of the Hopf algebra $\mathbb{k}\left[t^{ \pm 1}\right]$, special cases of the Hopf Ore construction which we review in the next subsection. 
2.3. Hopf Ore extensions and IHOEs. Hopf Ore extensions of a Hopf algebra $A$, denoted by $A[x ; \sigma, \delta]$, were defined and studied by Panov [Pan03] in 2003. His definition was refined and extended in [BO15] and then in Hu19, to iterated Hopf Ore extensions (IHOEs) over a field $\mathbb{k}$. The following definition is significantly more general than the original one given in Pan03, Definition 1.0] and its modification in BO15, Definition 2.1]. The improvement here is due to recent work of Huang Hu19. Recall that, given an algebra automorphism $\sigma$ of $A$, a $\sigma$-derivation $\delta$ of $A$ is a $\mathbb{k}$-linear endomorphism of $A$ such that

$$
\delta(a b)=\sigma(a) \delta(b)+\delta(a) b
$$

for all $a, b \in A$.

Definition 2.7. Let $\mathbb{k}$ be a field.

(1) Let $A$ be a Hopf $\mathbb{k}$-algebra. A Hopf Ore extension (abbreviated to HOE) of $A$ is an algebra $H$ such that

(1a) $H$ is a Hopf $\mathbb{k}$-algebra with Hopf subalgebra $A$;

(1b) there exist an algebra automorphism $\sigma$ of $A$ and a $\sigma$-derivation $\delta$ of $A$ such that $H=A[x ; \sigma, \delta]$.

(2) An (n-step) iterated Hopf Ore extension of $\mathbb{k}$ (abbreviated to (n-step) IHOE $($ of $\mathbb{k})$ ) is a Hopf algebra

$$
H=\mathbb{k}\left[X_{1}\right]\left[X_{2} ; \sigma_{2}, \delta_{2}\right] \cdots\left[X_{n} ; \sigma_{n}, \delta_{n}\right],
$$

where

(2a) $H$ is a Hopf algebra;

(2b) $H_{(i)}:=\mathbb{k}\left\langle X_{1}, \ldots, X_{i}\right\rangle$ is a Hopf subalgebra of $H$ for $i=1, \ldots, n$;

(2c) $\sigma_{i}$ is an algebra automorphism of $H_{(i-1)}$, and $\delta_{i}$ is a $\sigma_{i}$-derivation of $H_{(i-1)}$, for $i=2, \ldots, n$.

The definition of an HOE in BO15, Definition 2.1] required, in addition to Definition 2.7 (1a,1b), that

(1c) there are $a, b \in A$ and $v, w \in A \otimes A$ such that

$$
\Delta(x)=a \otimes x+x \otimes b+v(x \otimes x)+w .
$$

However, by Huang's theorem [Hu19, Theorem 1.3], if $A \subseteq H$ are noetherian $\mathbb{k}$ algebras satisfying hypotheses (1a) and (1b) of Definition 2.7(1), and $A \otimes A$ is a domain, then, up to a change of variable,

(1d) there are $a \in A$ and $w \in A \otimes A$ such that

$$
\Delta(x)=a \otimes x+x \otimes 1+w .
$$

This means that under the conditions in [Hu19, Theorem 1.3], namely that $A \otimes A$ is a noetherian domain, Definition 2.7(1) is equivalent to BO15, Definition 2.1]. For the subalgebras $H_{(i)}$ appearing in Definition 2.7(2) it is easy to show that $H_{(i)} \otimes H_{(i)}$ is a noetherian domain, so a similar comment applies to the comultiplication of an IHOE over $\mathbb{k}$. There are moreover strong restrictions on the automorphisms $\sigma_{i}$ and derivations $\delta_{i}$ which can occur - for details, see [BO15, Hu19] or [BZ20].

In Examples 2.8 and 2.9 in the next subsection we construct Hopf Ore extensions that are generalizations of the Hopf Ore extensions $A(n, q)$ and $C(n)$ of Theorem 2.3 . 
2.4. Non-noetherian Hopf domains of GK-dimension 2. In this subsection we describe two families of Hopf domains of GK-dimension two which are not affine and not noetherian.

Example 2.8. GZ17, Example 1.2] Let $K=\mathbb{k} G$ where $G$ is a group, let $\chi: G \rightarrow$ $\mathbb{k}^{\times}$be a character of $G$ and choose any element $e$ in the center of $G$, denoted by $Z(G)$. Define an algebra automorphism $\sigma_{\chi}: K \rightarrow K$ by

$$
\sigma_{\chi}(g)=\chi(g) g, \quad \forall g \in G,
$$

so $\sigma_{\chi}$ is the winding automorphism associated to the algebra map from $K$ to $\mathbb{k}$ induced by $\chi$. Let $\delta=0$. By WZZ16, Example 5.4], $K\left[z ; \sigma_{\chi}\right]$ is an HOE of $K$ with $\Delta(z)=z \otimes 1+e \otimes z$. This HOE is denoted by $A_{G}(e, \chi)$.

Now carry out the above construction when $G$ is a non-trivial non-cyclic subgroup of $(\mathbb{Q},+)$. Then

$$
\mathrm{GKdim} \mathbb{k} G=1, \quad \text { and } \quad \operatorname{GKdim} A_{G}(e, \chi)=2,
$$

using [HK96, Lemma 2.2] for the second equality. By [GZ17, Theorem 0.1(4)], $A_{G}(e, \chi)$ satisfies E2.1.1). When, for instance, $\mathbb{k}=\mathbb{C}$, there are many characters of $G$; for example, with $\lambda \in \mathbb{R}, \exp _{\lambda}: r \rightarrow \exp (2 \pi i r \lambda)$ is a character from $(\mathbb{Q},+)$ to $\mathbb{C}^{\times}$. Since $A_{G}(e, \chi)$ is a free $\mathbb{k} G$-module it is clear that $A_{G}(e, \chi)$ is not noetherian. Being a skew polynomial algebra with coefficient ring the group algebra of a torsionfree abelian group, $A_{G}(e, \chi)$ is a domain.

Example 2.9. GZ17, Example 1.3] Again, let $K=\mathbb{k} G$ where $G$ is a group and fix $e \in Z(G)$. Let $\tau: G \rightarrow(\mathbb{k},+)$ be an additive character of $G$. Define a $\mathbb{k}$-linear derivation $\delta: K \rightarrow K$ by

$$
\delta(g)=\tau(g) g(e-1), \quad \forall g \in G .
$$

Then $C_{G}(e, \tau):=K[z ; \delta]$ is an HOE of $K$ when we define $\Delta(z)=z \otimes 1+e \otimes z$.

As in the previous example, let $G$ be a nontrivial non-cyclic subgroup of $(\mathbb{Q},+)$. Again by [HK96, Lemma 2.2] (or [Zha97, Theorem 1.2(1)])

$$
\operatorname{GKdim} \mathbb{k} G=1, \quad \text { and } \quad \operatorname{GKdim} C_{G}(e, \tau)=2 .
$$

Since we are assuming $\mathbb{k}$ has characteristic zero, there are many additive characters from $G$ to $(\mathbb{k},+)$. For example, let $\lambda \in \mathbb{k}$; then $i_{\lambda}: r \rightarrow r \lambda$ is an additive character from $G \rightarrow(\mathbb{k},+)$.

Denote the augmentation ideal of $K$ by $K^{+}$. Since $C_{G}(e, \tau) K^{+}$is an ideal of $C_{G}(e, \tau)$ with $C_{G}(e, \tau) / C_{G}(e, \tau) K^{+} \cong \mathbb{k}[z], C_{G}(e, \chi)$ satisfies (E2.1.1). In the same way as Example 2.8, $C_{G}(e, \tau)$ is not noetherian, but it is a domain.

The next family of examples are not HOEs, although they are like the others assembled from two domains of GK-dimension 1. Since the details are quite technical, we only give a brief sketch here, referring the reader to GZ17] for more information.

Example 2.10. GZ17, Construction 3.1] Let $G$ be a nontrivial non-cyclic subgroup of $(\mathbb{Q},+)$, let $\left\{p_{i}: i \in I\right\}$ be an infinite set of pairwise relatively prime integers, and let $\chi$ be a character of a certain group $G M$ with $G \subseteq G M \subseteq(\mathbb{Q},+)$. Given this data one can construct (over several pages) a non-affine Hopf domain of GKdimension two, denoted by $B_{G}\left(\left\{p_{i}\right\}, \chi\right)$. By [GZ17, Construction 1.2], $B_{G}\left(\left\{p_{i}\right\}, \chi\right)$ satisfies (E2.1.1) and is not noetherian.

Here is the classification result parallel to Theorem 2.3 , Let $\mathbb{Z}_{\langle 2\rangle}$ denote the localization of the ring $\mathbb{Z}$ at the maximal ideal $\langle 2\rangle$. 
Theorem 2.11. GZ17, Theorem 0.1] Assume that $\mathbb{k}$ has characteristic 0 and let $H$ be a Hopf domain over $\mathbb{k}$ of GK-dimension two which is not noetherian and satisfies (E2.1.1). Then $H$ is isomorphic as a Hopf algebra to one of the following.

(1) $\mathbb{k} G$ where $G$ is a non-finitely generated subgroup of $\mathbb{Q}^{2}$ containing $\mathbb{Z}^{2}$.

(2) $\mathbb{k} G$ where $G=L \rtimes R$ for subgroups $L$ of $\mathbb{Q}$ containing $\mathbb{Z}$ and $R$ of $\mathbb{Z}_{(2)}$ containing $\mathbb{Z}$, with at least one of $L$ and $R$ not finitely generated.

(3) $A_{G}(e, \chi)$ in Example 2.8

(4) $C_{G}(e, \tau)$ in Example 2.9.

(5) $B_{G}\left(\left\{p_{i}\right\}, \chi\right)$ in Example 2.10

Thus Theorems 2.3 and 2.11 together with Lemma 1.1 and Remark 1.2(1) give a complete classification when $\mathbb{k}$ has characteristic 0 of the Hopf $\mathbb{k}$-algebra domains of GK-dimension at most 2 which satisfy (E2.1.1). But not all Hopf domains of GK-dimension 2 satisfy (E2.1.1), as we explain in the next subsection.

2.5. Hopf domains of GK-dimension 2 with no infinite classical subgroup. The hope that the algebras of Subsection 2.2 constitute a complete list of affine Hopf domains of GK-dimension 2 is demolished by the following result. As the notation suggests, the new algebras are variants of those in Examples 2.5 .

Theorem 2.12. WZZ13 Assume that $\mathbb{k}$ has characteristic 0 . Let $s, p_{1}, \ldots, p_{s}$ be integers, all greater than 1 and with $\operatorname{gcd}\left(p_{i}, p_{j}\right)=1$ when $i \neq j$. Fix scalars $\sigma_{1}, \ldots, \sigma_{s}$ in $\mathbb{k}$, not all equal. For $\ell:=\prod_{i=1}^{s} p_{i}$, choose $q$, an $\ell$ th root of 1 in $\mathbb{k}$. Then there is a family $B\left(n,\left\{p_{i}\right\}_{1}^{s}, q,\left\{\sigma_{i}\right\}_{1}^{s}\right)$ of Hopf $\mathbb{k}$-algebras with the following properties.

(1) $B\left(n,\left\{p_{i}\right\}_{1}^{s}, q,\left\{\sigma_{i}\right\}_{1}^{s}\right)$ is an affine domain of GK-dimension 2.

(2) $B\left(n,\left\{p_{i}\right\}_{1}^{s}, q,\left\{\sigma_{i}\right\}_{1}^{s}\right)$ does not satisfy (E2.1.1).

(3) $B\left(n,\left\{p_{i}\right\}_{1}^{s}, q,\left\{\sigma_{i}\right\}_{1}^{s}\right)$ is pointed, generated by grouplikes and skew primitives.

(4) $B\left(n,\left\{p_{i}\right\}_{1}^{s}, q,\left\{\sigma_{i}\right\}_{1}^{s}\right)$ is a finite module over its affine center, hence is noetherian and satisfies a polynomial identity.

(5) $\operatorname{gldim} B\left(n,\left\{p_{i}\right\}_{1}^{s}, q,\left\{\sigma_{i}\right\}_{1}^{s}\right)<\infty$ if and only if $s=2$ and $\sigma_{1} \neq \sigma_{2}$. In this case the global dimension is 2.

(6) Up to a permutation of $\{1, \ldots, s\}$ and multiplication of $\left\{\sigma_{i}\right\}_{1}^{s}$ by a non-zero scalar, these Hopf algebras are mutually non-isomorphic.

Wang, Zhang and Zhuang show in WZZ13 that any as yet unknown example of an affine Hopf domain of GK-dimension 2 in characteristic 0, especially if generated by grouplikes and skew primitives, would have to exhibit very constrained features. They therefore ask:

Question 2.13. WZZ13, Remark 4.5] Assume $\mathbb{k}$ has characteristic 0. Are the algebras of Theorem 2.12 the only affine Hopf $\mathbb{k}$-algebra domains of GK-dimension 2 not satisfying (E2.1.1)?

It is tempting, given the classification in characteristic 0 of prime AS-regular algebras of dimension 1 and the partial classification of GK-dimension 2 Hopf domains, to consider prime AS-regular Hopf algebras of GK-dimension 2. Proposition 1.17 indicates that this may be a formidable task, so perhaps a preliminary step might be the following.

Question 2.14 (Dingguo Wang Wa20]). Find homological invariants (additional to, or generalising, the integral order and integral minor) which are defined for 
pointed Hopf algebras, and which may help to classify prime pointed Hopf algebras of GK-dimension two.

2.6. 2-step IHOEs in positive characteristic. In this subsection let $p$ be a prime and let $\mathbb{k}$ be an algebraically closed field of characteristic $p$. Consider the project to classify the affine Hopf $\mathbb{k}$-algebra domains of GK-dimension at most 2 . If $H$ is such an algebra with GKdim $H=1$, then $H$ is commutative by Tsen's theorem Co77, p. 374], and so $H=\mathbb{k}[t]$ or $\mathbb{k}\left[t^{ \pm 1}\right]$ by Lemma 1.1.

However when GKdim $H=2$ the situation appears much more complex than in characteristic 0 . The connected Hopf $\mathbb{k}$-algebras, that is those whose coradical is $\mathbb{k}$, form a good starting point. In arbitrary characteristic every IHOE is connected by [BO15, Proposition 2.5]. The cocommutative connected Hopf algebras in characteristic 0 are the enveloping algebras of Lie algebras. Zhuang showed in Zhu13, Proposition 7.6] that in characteristic 0, there are only two connected Hopf algebras of GK-dimension 2, and both are cocommutative IHOEs: namely the enveloping algebras of the two 2-dimensional Lie algebras. But in positive characteristic it's a different story, as we now explain.

Let $\mathbf{d}_{\mathbf{s}}=\left\{d_{s}\right\}_{s \geq 0}, \mathbf{b}_{\mathbf{s}}=\left\{b_{s}\right\}_{s \geq 0}$ and $\mathbf{c}_{\mathbf{s}, \mathbf{t}}=\left\{c_{s, t}\right\}_{0 \leq s<t}$ be sequences of scalars in $\mathbb{k}$ with only finitely many nonzero terms. Let $H\left(\mathbf{d}_{\mathbf{s}}, \mathbf{b}_{\mathbf{s}}, \mathbf{c}_{\mathbf{s}, \mathbf{t}}\right)$ denote the Ore extension $\mathbb{k}\left[X_{1}\right]\left[X_{2} ; \mathrm{Id}, \delta\right]$ where

$$
\delta\left(X_{1}\right)=\sum_{s \geq 0} d_{s} X_{1}^{p^{s}}
$$

and where the coalgebra structure is as follows. Define maps $\Delta, \epsilon$, and $S$ on $\left\{X_{1}, X_{2}\right\}$ by setting

$$
\begin{aligned}
\Delta\left(X_{1}\right) & =X_{1} \otimes 1+1 \otimes X_{1}, \\
\Delta\left(X_{2}\right) & =X_{2} \otimes 1+1 \otimes X_{2}+w, \\
\epsilon\left(X_{1}\right) & =\epsilon\left(X_{2}\right)=0, \\
S\left(X_{1}\right) & =-X_{1}, \\
S\left(X_{2}\right) & =-X_{2}-m(\operatorname{Id} \otimes S)(w),
\end{aligned}
$$

where

$$
\begin{aligned}
w=\sum_{s \geq 0} b_{s} & \left(\sum_{i=1}^{p-1} \frac{(p-1) !}{i !(p-i) !}\left(X_{1}^{p^{s}}\right)^{i} \otimes\left(X_{1}^{p^{s}}\right)^{p-i}\right) \\
& +\sum_{0 \leq s<t} c_{s, t}\left(X_{1}^{p^{s}} \otimes X_{1}^{p^{t}}-X_{1}^{p^{t}} \otimes X_{1}^{p^{s}}\right) .
\end{aligned}
$$

Theorem 2.15. BZ20, Theorem 0.3(3)] Let $\mathbb{k}$ be algebraically closed of characteristic $p>0$ and let $H$ be a 2-step IHOE over $\mathbb{k}$.

(1) The Hopf algebra $H$ is isomorphic to $H\left(\mathbf{d}_{\mathbf{s}}, \mathbf{b}_{\mathbf{s}}, \mathbf{c}_{\mathbf{s}, \mathbf{t}}\right)$ for some choice of scalars $\left\{d_{s}\right\}_{s \geq 0},\left\{b_{s}\right\}_{s \geq 0}$ and $\left\{c_{s, t}\right\}_{0 \leq s<t}$.

(2) Two such Hopf algebras $H\left(\mathbf{d}_{\mathbf{s}}, \mathbf{b}_{\mathbf{s}}, \mathbf{c}_{\mathbf{s}, \mathbf{t}}\right)$ and $H\left(\left(\mathbf{d}_{\mathbf{s}}^{\prime}, \mathbf{b}_{\mathbf{s}}^{\prime}, \mathbf{c}_{\mathbf{s}, \mathbf{t}}^{\prime}\right)\right.$ are isomorphic as Hopf algebras if and only if there are nonzero scalars $\alpha, \beta \in \mathbb{k}$ such that

$$
d_{s}^{\prime}=d_{s} \alpha^{p^{s}-1} \beta^{-1}, \quad b_{s}^{\prime}=b_{s} \alpha^{p^{s+1}} \beta^{-1}, \quad c_{s, t}^{\prime}=c_{s, t} \alpha^{p^{s}+p^{t}} \beta^{-1}
$$

for all $s, t$.

The connected unipotent $\mathbb{k}$-groups $U$ of dimension $n$ are precisely those algebraic groups whose coordinate ring is an $n$-step commutative IHOE over $\mathbb{k}$, as explained in 
BZ20, §6.1]. Thus Theorem2.15incorporates the classification of the 2-dimensional connected unipotent groups over $\mathbb{k}$ - namely, their coordinate rings are the algebras $H\left(\mathbf{0}, \mathbf{b}_{\mathbf{s}}, \mathbf{c}_{\mathbf{s}, \mathbf{t}}\right)$. The classification in terms of the groups rather than their coordinate rings can be found in [KMT74, §3.7]. For further information concerning the algebras $H\left(\mathbf{d}_{\mathbf{s}}, \mathbf{b}_{\mathbf{s}}, \mathbf{c}_{\mathbf{s}, \mathbf{t}}\right)$, see BZ20].

As suggested above, one should view Theorem 2.15 as the first step in a program to classify the connected affine Hopf domains of GK-dimension 2 over $\mathbb{k}$ when $\mathbb{k}$ has positive characteristic. From this perspective and in the light of Zhuang's classification in characteristic 0, Zhu13, Proposition 7.6], it is natural to ask:

Question 2.16. BZ20, Question 6.3] Is every affine connected Hopf domain over $\mathbb{k}$ of GK-dimension 2 an IHOE?

\section{Further COMments, PROJECTS AND OPEN QUESTIONS}

In this final section $\mathbb{k}$ denotes an arbitrary algebraically closed field unless otherwise stated. We review the questions scattered throughout the previous sections and gather them into a few overarching projects, and then suggest a couple of further directions of research. All these questions and projects should be viewed as supplementing (and in some cases repeating) questions in other survey articles, for example [And14, And17, Br98, Br07, BGi14, BGLZ14, Go13, KWZ19.

First we note the familiar questions reappearing in this paper which concern the entire category of affine Hopf algebras of finite GK-dimension, namely Question 0.1 and Conjecture 0.2. To these we add the following, which has been implicit at several points in the foregoing pages.

Question 3.1. Is every affine Hopf algebra of finite GK-dimension noetherian?

The only evidence in favor of a positive answer to Question 3.1 seems to be the absence of counterexamples. The converse is false - consider for example the group algebra of a polycyclic group with is not nilpotent-by-finite. It does however seem to be unknown whether a noetherian Hopf algebra has to be affine.

3.1. Projects in GK-dimensions one and two. The questions listed earlier concerning Hopf algebras of GK-dimension 1 are Questions 1.13, 1.14, 1.15, and 1.16. These should be regarded as key components on the road towards:

Project 3.2. Classify semiprime noetherian Hopf $\mathbb{k}$-algebras of GK-dimension one.

Question 1.18 focuses on the possibility of a close link between GK-dimensions one and two. It would be answered as an immediate corollary of a classification result in GK-dimension two.

The target of classifying all affine or noetherian semiprime (or even prime) Hopf algebras of GK-dimension two seems rather too ambitious at present, so we propose:

Project 3.3. Classify Hopf $\mathbb{k}$-algebra domains of GK-dimension two.

Additional hypotheses to impose as milestones on the route to Project 3.3 include those of Question 2.13- namely that $\mathbb{k}$ has characteristic 0 and the algebra is affine; and those of Question 2.16, that $\mathbb{k}$ has positive characteristic and the Hopf algebra is affine and connected. 
3.2. Beyond GK-dimension two. The following special case of Question 0.1 is currently open:

Question 3.4. Does there exist a Hopf $\mathbb{k}$-algebra $H$ with $2<\operatorname{GKdim} H<3$ ?

Strong evidence in support of a positive answer to this question is given by [WZZ16, Theorem 0.1], which confirms that the answer is "No" when $\mathbb{k}$ has characteristic 0 , with $H$ affine and pointed. In the light of their result Wang, Zhang and Zhuang proposed the following, suggesting that double Ore extensions may be an important tool for this purpose:

Project 3.5. WWZ16, Remark 7.5] In characteristic 0, classify affine pointed Hopf domains of GK-dimension three.

3.3. Connected (graded) Hopf algebras. Observe that if the pointed hypothesis in Project 3.5 is strengthened to require that the Hopf algebra is connected, the proposed classification has been achieved by Zhuang [Zhu13, and even in dimension four in WZZ15. As these results suggest, the class of connected Hopf algebras, and even the smaller classes of those which are graded as algebras and/or as coalgebras, form interesting testing grounds.

For example, in BGZ19], Brown, Gilmartin and Zhang studied, for $\mathbb{k}$ of characteristic 0 , Hopf $\mathbb{k}$-algebras of finite GK-dimension that are connected graded as an algebra. In particular, they proved that such a Hopf algebra is a noetherian domain which is GK-Cohen-Macaulay, AS regular and Auslander regular of global dimension equal to its GK-dimension, and is Calabi-Yau. As a consequence, it satisfies Poincaré duality.

Remaining in characteristic 0 , but imposing the stronger hypothesis on the Hopf algebra $H$ of finite GK-dimension, that it is connected graded both as an algebra and as a coalgebra, Zhou, Shen and Lu proved the striking result [ZSL19 that $H$ is an IHOE [ZSL19, Theorem B]. Naturally, this leads to

Question 3.6. ZZSL19, Question C] With $\mathbb{k}$ of characteristic 0, suppose the Hopf $\mathbb{k}$-algebra $H$ has finite GK-dimension and is connected graded as an algebra. Is $\mathrm{H}$ an IHOE?

Acknowledgments. The authors thank the editors for the invitation to provide this submission, and thank Professors Gongxiang Liu and Dingguo Wang for several conversations on the subject, for reading an early draft of the paper, for suggesting some projects. K.A. Brown was partially supported by Leverhulme Emeritus Fellowship (EM-2017-081-9) and J.J. Zhang by the US National Science Foundation (No. DMS-1700825).

\section{REFERENCES}

[And14] N. Andruskiewitsch, On finite-dimensional Hopf algebras, Proceedings of the International Congress of Mathematicians, Seoul (2014) Vol. II, 117-141, Kyung Moon Sa, Seoul, 2014.

[And17] N. Andruskiewitsch, An introduction to Nichols algebras, Quantization, geometry and noncommutative structures in mathematics and physics, 135-195, Math. Phys. Stud., Springer, 2017.

[AA06] N. Andruskiewitsch and I.E. Angiono, On Nichols algebras with generic braiding, in Modules and Comodules (Porto 2006) (Brzeziński T., et al., Editors) (Birkhäuser Verlag, Basel, 2008), 47-64. 
[AAH19] N. Andruskiewitsch, I.E. Angiono and I. Heckenberger, On finite GK-dimensional Nichols algebras of diagonal type. Tensor categories and Hopf algebras, 1-23, Contemp. Math., 728, Amer. Math. Soc., Providence, RI, 2019.

[AS00] N. Andruskiewitsch and H.-J. Schneider, Finite quantum groups and Cartan matrices, Adv. Math. 154 (2000), 1-45.

[AS04] N. Andruskiewitsch and H.-J. Schneider, A characterization of quantum groups, J. Reine Angew. Math. 577 (2004), 81-104.

[Ang13] I.E. Angiono, On Nichols algebras of diagonal type, J. Reine Angew. Math. 683 (2013), 189-251.

[Bo91] A. Borel, Linear Algebraic Groups, second enlarged ed., Springer-Verlag, New York, 1991.

[Br98] K.A. Brown, Representation theory of Noetherian Hopf algebras satisfying a polynomial identity, in Trends in the representation theory of finite dimensional algebras (Seattle 1997) (Green E. L. and Huisgen-Zimmermann B., Editors), Contemporary Mathematics, Vol. 229 (AMS, Providence, RI, 1998), 49-79.

[Br07] K.A. Brown, Noetherian Hopf algebras, Turkish J. Math. 31 (2007), suppl., 7-23.

[BGi14] K.A. Brown and P. Gilmartin, Hopf algebras under finiteness conditions, Palest. J. Math. 3 (2014), Special issue, 356-365.

[BGZ19] K.A. Brown, P. Gilmartin and J.J. Zhang, Connected (graded) Hopf algebras, Trans. Amer. Math. Soc. 372 (2019), no. 5, 3283-3317.

[BG97] K.A. Brown and K.R. Goodearl, Homological aspects of Noetherian PI Hopf algebras and irreducible modules of maximal dimension, J. Algebra 198 (1997), 240-265.

[BGLZ14] K.A. Brown, K.R. Goodearl, T. Lenagan and J.J. Zhang, Mini-Workshop: infinite dimensional Hopf algebras, Abstracts from the mini-workshop held April 1319, 2014, Oberwolfach Rep. 11 (2014), no. 2, 1111-1137.

[BM17] K.A. Brown and M.J. Macleod, The Cohen Macaulay property for noncommutative rings Algebr. Represent. Theory 20 (2017), no. 6, 14331465.

[BO15] K.A. Brown, S. O'Hagan, J.J Zhang, G. Zhuang, Connected Hopf algebras and iterated Ore extensions, J. Pure Appl. Algebra, 219 (2015), no. 6, 2405-2433.

[BZ08] K.A. Brown and J.J. Zhang, Dualising complexes and twisted Hochschild (co)homology for Noetherian Hopf algebras, J. Algebra 320 (2008), no. 5, 1814-1850.

[BZ10] K.A. Brown and J.J. Zhang, Prime regular Hopf algebras of GK-dimension one, Proc. Lond. Math. Soc. (3) 101 (2010), no. 1, 260-302.

[BZ20] K.A. Brown and J.J. Zhang, Iterated Hopf Ore Extensions in positive characteristic, in preparation, 2020.

[Co77] P.M. Cohn, Algebra. Vol. 2. With errata to Vol. I. John Wiley \& Sons, London-New YorkSydney, 1977.

[EGNO15] P. Etingof, Pavel. S. Gelaki, D. Nikshych, V. Ostrik, Tensor categories Mathematical Surveys and Monographs, 205, Amer. Math. Soc., Providence, RI, 2015.

[Go13] K.R. Goodearl, Noetherian Hopf algebras, Glasgow Math. J. 55 (2013), 75-87.

[GZ10] K.R. Goodearl, J.J. Zhang, Noetherian Hopf algebra domains of Gelfand-Kirillov dimension two, J. Algebra, 324 (2010), 3131-3168.

[GZ17] K.R. Goodearl, J.J. Zhang, Non-affine Hopf algebra domains of Gelfand-Kirillov dimension two, Glasgow Math. J. 59 (2017), no. 3, 563-593.

[Hu19] H.-D. Huang, Hopf Ore extensions, preprint, (2019) arXiv:1902.02237

[HK96] C. Huh and C.O. Kim, Gelfand-Kirillov dimension of skew polynomial rings of automorphism type, Comm. Algebra 24 (1996), 2317-2323.

[Hu75] J.E. Humphreys, Linear Algebraic Groups, Graduate Texts in Mathematics, 21, SpringerVerlag, New York-Heidelberg, 1975.

[KMT74] T. Kambayashi, M. Miyanishi and M. Takeuchi, Unipotent Algebraic Groups, Lecture Notes in Math. 414, Springer, 1974.

[KL00] G.R. Krause and T.H. Lenagan, Growth of algebras and Gelfand-Kirillov dimension, Revised edition, Graduate Studies in Mathematics, 22, Amer. Math. Soc., 2000.

[KWZ19] H. Krause, S. Witherspoon and J.J. Zhang, Mini-Workshop: Cohomology of Hopf Algebras and Tensor Categories, Oberwolfach Rep. 16 (2019), no. 1, 663-693.

[Li09] G. Liu, On Noetherian affine prime regular Hopf algebras of Gelfand-Kirillov dimension 1, Proc. Amer. Math. Soc. 137 (2009), no. 3, 777-785.

[Li20] G. Liu, A classification result on prime Hopf algebras of GK-dimension one, J. of Algebra, $\mathbf{5 4 7}$ (2020), 579-667. 
[Li20a] G. Liu, Oral communication, (2020).

[LWZ07] D.-M. Lu, Q.-S. Wu and J.J. Zhang, Homological integral of Hopf algebras, Trans. Amer. Math. Soc. 359 (2007), 49454975.

[LWZ09] D.-M. Lu, Q.-S. Wu and J.J. Zhang, Hopf algebras with rigid dualizing complexes, Israel J. Math. 169 (2009), 89-108.

[Mo93] S. Montgomery, Hopf Algebras and their Actions on Rings, CBMS Regional Conference Series in Mathematics 82 (AMS, Providence, 1993).

[Pan03] A.N. Panov, Ore extensions of Hopf algebras, Mat. Zametki, 74 (2003), 425-434.

[Pas77] D.S. Passman, The Algebraic Structure of Group Rings, Wiley-Interscience, 1977.

[SW84] L.W. Small and R.B. Warfield, Jr., Prime affine algebras of Gelfand-Kirillov dimension one, J. Algebra 91 (1984), no. 2, 386389.

[SSW85] L.W. Small, J.T. Stafford, R.B. Warfield, R. B., Affine algebras of Gel'fand-Kirillov dimension one are PI, Math. Proc. Camb. Phil. Soc. 97 (1985), no. 3, 407414.

[Ta71] E.J. Taft, The order of the antipode of finite-dimensional Hopf algebra, Proc. Nat. Acad. Sc. 68 (1971), 2631-2633.

[Wa20] D.-G. Wang, Oral communication, (2020).

[WZZ13] D.-G. Wang, J.J. Zhang and G. Zhuang, Hopf algebras of GK-dimension two with vanishing Ext-group, J. Algebra 388 (2013) 219247.

[WZZ15] D.-G. Wang, J.J. Zhang and G. Zhuang, Connected Hopf algebras of Gelfand-Kirillov dimension four, Trans. Amer. Math. Soc. 367 (2015) 5597-5632.

[WZZ16] D.-G. Wang, J.J. Zhang and G. Zhuang, Primitive cohomology of Hopf algebras, J. Algebra 464 (2016), 36-96.

[WLD16] J.-Y. Wu, G.-X. Liu and N.-Q. Ding, Classification of affine prime regular Hopf algebras of GK-dimension one, Adv. Math. 296 (2016), 1-54.

[WZ03] Q.-S. Wu and J. J. Zhang, Noetherian PI Hopf algebras are Gorenstein, Trans. Amer. Math. Soc. 355 (2003) 1043-1066.

[ZSL19] G.-S. Zhou, Y. Shen and D.-M. Lu, The structure of connected (graded) Hopf algebras, preprint, (2019) arXiv:1904.01918

[Zha97] J.J. Zhang, A note on GK dimension of skew polynomial extensions, Proc. Amer. Math. Soc. 125 (1997), no. 2, 363-373.

[Zhu13] G.-B. Zhuang, Properties of pointed and connected Hopf algebras of finite GelfandKirillov dimension, J. London Math. Soc. (2) 87 (2013), 877-898.

School of Mathematics and Statistics, University of Glasgow, Glasgow G12 8QW, SCOTLAND

E-mail address: ken.brown@glasgow.ac.uk

Department of Mathematics, Box 354350, University of Washington, Seattle, WA 98195, USA

E-mail address: zhang@math.washington.edu 